\title{
Analysis of al-2 Mutations in Neurospora
}

Violeta Díaz-Sánchez, Alejandro F. Estrada, Danika Trautmann, M. Carmen Limón, Salim Al-Babili and Javier Avalos

\section{Supporting information 1}

\author{
al-2 sequences in mutant strains of $N$. crassa \\ Start and stop codons in bold letters (increased font size) \\ Introns in red lower case \\ Mutations underlined and shaded in color
}

Wild-type al-2 allele (from Accession No. L27652)

\begin{abstract}
ATGTACGACTATGCTTTTGTgtgagtaccagatctgacagccaccaccctttagtcctgggaaacatcattgctgatga gatacatgatagTCACTTAAAATTCACGGTACCCGCGGCGGTACTTCTCACCGCTATCGCCTACCCCATTCTCAACAGGA TACATCTCATCCAAACAGGCTTCCTCGTCGTCGTCGCCTTTACCGCCGCTCTGCCATGGGATGCCTACTTGATTAAGCAC AAAGTATGGTCTTACCCACCAGAAGCCATTGTTGGGCCGCGTTTGCTTGGAATTCCCTTTGAAGAGCTGTTCTTCTTTGT GATACAGACTTACATCACGGCGCTCGTATACATCCTCTTCAACAAGCCGGTGCTGCACGCGTTGCACCTCAACAATCAAC AAAACCCGCCAGCATGGATGAGGGTTGTCAAGGTTACCGGCCAGGTAGTCCTCGTAGCCTTGTCGGTATGGGGATGGAAT GCCGCTCAGGTTCATCAGGAAACAAGCTATCTCGGCTTGATCCTTGTTTGGGCTTGTCCGTTCTTACTGGCTATCTGGAC ССTCGCTGGGCGCTTCATTCTCAGCCTACCCTGGTACGCGACGGTGCTCCCGATGTTCCTACCCACCTTCTATCTTTGGG CGGTAGACGAGTTTGCCTTGCACAGGGGTACTTGGTCCATCGGATCGGGGACGAAGCTCGATTTTTGTCTGTTTGGCAAG TTGGACATTGAAGAAGCCACGTTCTTCCTGGTGACCAACATGCTCATCGTTGGCGGTATGGCCGCGTTCGATCAATATCT GGCCGTCATTTACGCTTTCCCAACTCTGTTCCCCAAGGTCAACCGGTATCCGACAACTCATATGCTTCTTCAAAGCCGTC TTATCAACACTTCCAGGTACGATCTTGAGCGCATTGAGGGCCTGAGAGAAGCGGTCGAGAGACTGCGCCTGAAGAGCAGG AGTTTTTACCTGGCCAATTCGCTCTTTTCTGGTCGACTCCGCATTGACCTGATCCTGCTgtaagtacccattcagcCtaa gcaagttccggacttgaactaataatcatctcagGTACTCCTTCTGTCGCCTGGCTGATGATCTAGTCGACGACGCCA AATCTCGCCGTGAGGTCTTGTCCTGGACCGCGAAGCTGAACCACTTCCTTGATCTGCACTACAAGGACGCGGACGCCACC GAGGACCCCAAGAAAAAGGCGGAGCGAATCGACGCCTACATCAAGACAGCGTTCCCTCCCTGTGCCTACCAAGCCCTCCA ССTCCTGCCCACTCACATTCTTCCTCCCAAGCСTCTTTACGATCTCATCAAGGGTTTCGAGATGGACTCTCAATTCACCT TCCACGGTACTTCCGACTCTACGGATCTCCAATACCCCATCGCCGACGACAAGGACCTTGAGAACTACGCTATCTATGTC GCCGGTACCGTCGGCGAGCTCTGCATCGCCCTCATCATCTACCACTGCCTGCCAGACATGTCGGACACTCAGAAGCGCGA GCTCGAGACCGCCGCGTGCCGGATGGGCATCGCGCTGCAGTACGTCAACATCGCTCGTGACATCGTCGTCGACGCACGTA TCGGGCGCGTTTACTTGCCTACCACCTGGCTCAAGAAGGAAGGGTTGACGCACAAGATGGTCTTGGAGAACCCCGAGGGT CCCGAGGTCATTGAGCGGATGAGAAGACGGCTTTTGGAAAATGCGTTTGAGCTGTATGGGGGCGCGAGGCCTGAGATGCA ACGGATACCGAGCGAGGCTAGGGGCCCGATGATTGGTGCCGTTGAAAATTACATGGCGATTGGAAGGGTGTTGAGGGAGA GGAAGGAGGGGACGGTGTTTGTGAGGATGGAGGGGAGGGCTACGGTCCCGAAGCGAAGGAGGTTGAGCACGCTGTTGAGG GCGTTGTATGAGCAGTAG
\end{abstract}




\section{Mutant alleles}

FGSC896 (Accession No. FR745430)

Replacement of gt by $\underline{A A}$; intron splicing site is changed (see text)

ATGTACGACTATGCTTTTGTgtgagtaccagatctgacagccaccaccctttagtcctgggaaacatcattgctgatga gatacatgatagTCACTTAAAATTCACGGTACCCGCGGCGGTACTTCTCACCGCTATCGCCTACCCCATTCTCAACAGGA TACATCTCATCCAAACAGGCTTCCTCGTCGTCGTCGCCTTTACCGCCGCTCTGCCATGGGATGCCTACTTGATTAAGCAC AAAGTATGGTCTTACCCACCAGAAGCCATTGTTGGGCCGCGTTTGCTTGGAATTCCCTTTGAAGAGCTGTTCTTCTTTGT GATACAGACTTACATCACGGCGCTCGTATACATCCTCTTCAACAAGCCGGTGCTGCACGCGTTGCACCTCAACAATCAAC AAAACCCGCCAGCATGGATGAGGGTTGTCAAGGTTACCGGCCAGGTAGTCCTCGTAGCCTTGTCGGTATGGGGATGGAAT GCCGCTCAGGTTCATCAGGAAACAAGCTATCTCGGCTTGATCCTTGTTTGGGCTTGTCCGTTCTTACTGGCTATCTGGAC ССTCGCTGGGCGCTTCATTCTCAGCCTACCCTGGTACGCGACGGTGCTCCCGATGTTCCTACCCACCTTCTATCTTTGGG CGGTAGACGAGTTTGCCTTGCACAGGGGTACTTGGTCCATCGGATCGGGGACGAAGCTCGATTTTTGTCTGTTTGGCAAG TTGGACATTGAAGAAGCCACGTTCTTCCTGGTGACCAACATGCTCATCGTTGGCGGTATGGCCGCGTTCGATCAATATCT GGCCGTCATTTACGCTTTCCCAACTCTGTTCCCCAAGGTCAACCGGTATCCGACAACTCATATGCTTCTTCAAAGCCGTC TTATCAACACTTCCAGGTACGATCTTGAGCGCATTGAGGGCCTGAGAGAAGCGGTCGAGAGACTGCGCCTGAAGAGCAGG AGTTTTTACCTGGCCAATTCGCTCTTTTCTGGTCGACTCCGCATTGACCTGATCCTGCTAAAAgtacCcattcagcctaa gcaagttccggacttgaactaataaatcatctcagGTACTCCTTCTGTCGCCTGGCTGATGATCTAGTCGACGACGCC AAATCTCGCCGTGAGGTCTTGTCCTGGACCGCGAAGCTGAACCACTTCCTTGATCTGCACTACAAGGACGCGGACGCCAC CGAGGACCCCAAGAAAAAGGCGGAGCGAATCGACGCCTACATCAAGACAGCGTTCCCTCCCTGTGCCTACCAAGCCCTCC АССTCСTGCCСАСTCACATTCTTCCTCCCAAGCCTCTTTACGATCTCATCAAGGGTTTCGAGATGGACTCTCAATTCACC TTCCACGGTACTTCCGACTCTACGGATCTCCAATACCCCATCGCCGACGACAAGGACCTTGAGAACTACGCTATCTATGT CGCCGGTACCGTCGGCGAGCTCTGCATCGCCCTCATCATCTACCACTGCCTGCCAGACATGTCGGACACTCAGAAGCGCG AGCTCGAGACCGCCGCGTGCCGGATGGGCATCGCGCTGCAGTACGTCAACATCGCTCGTGACATCGTCGTCGACGCACGT ATCGGGCGCGTTTACTTGCCTACCACCTGGCTCAAGAAGGAAGGGTTGACGCACAAGATGGTCTTGGAGAACCCCGAGGG TCCCGAGGTCATTGAGCGGATGAGAAGACGGCTTTTGGAAAATGCGTTTGAGCTGTATGGGGGCGCGAGGCCTGAGATGC AACGGATACCGAGCGAGGCTAGGGGCCCGATGATTGGTGCCGTTGAAAATTACATGGCGATTGGAAGGGTGTTGAGGGAG AGGAAGGAGGGGACGGTGTTTGTGAGGATGGAGGGGAGGGCTACGGTCCCGAAGCGAAGGAGGTTGAGCACGCTGTTGAG GGCGTTGTATGAGCAGTAG

FGSC897 (Accession No. FR745431)

Insertion of CAAGA

ATGTACGACTATGCTTTTGTgtgagtaccagatctgacagccaccaccctttagtcctgggaacatcattgctgatga gatacatgatagTCACTTAAAATTCACGGTACCCGCGGCGGTACTTCTCACCGCTATCGCCTACCCCATTCTCAACAGGA TACATCTCATCCAAACAGGCTTCCTCGTCGTCGTCGCCTTTACCGCCGCTCTGCCATGGGATGCCTACTTGATTAAGCAC AAAGTATGGTCTTACCCACCAGAAGCCATTGTTGGGCCGCGTTTGCTTGGAATTCCCTTTGAAGAGCTGTTCTTCTTTGT GATACAGACTTACATCACGGCGCTCGTATACATCCTCTTCAACAAGCCGGTGCTGCACGCGTTGCACCTCAACAATCAAC AAAACCCGCCAGCATGGATGAGGGTTGTCAAGGTTACCGGCCAGGTAGTCCTCGTAGCCTTGTCGGTATGGGGATGGAAT GCCGCTCAGGTTCATCAGGAAACAAGCTATCTCGGCTTGATCCTTGTTTGGGCTTGTCCGTTCTTACTGGCTATCTGGAC ССTCGCTGGGCGCTTCATTCTCAGCCTACCCTGGTACGCGACGGTGCTCCCGATGTTCCTACCCACCTTCTATCTTTGGG CGGTAGACGAGTTTGCCTTGCACAGGGGTACTTGGTCCATCGGATCGGGGACGAAGCTCGATTTTTGTCTGTTTGGCAAG TTGGACATTGAAGAAGCCACGTTCTTCCTGGTGACCAACATGCTCATCGTTGGCGGTATGGCCGCGTTCGATCAATATCT GGCCGTCATTTACGCTTTCCCAACTCTGTTCCCCAAGGTCAACCGGTATCCGACAACTCATATGCTTCTTCAAAGCCGTC TTATCAACACTTCCAGGTACGATCTTGAGCGCATTGAGGGCCTGAGAGAAGCGGTCGAGAGACTGCGCCTGAAGAGCAGG AGTTTTTACCTGGCCAATTCGCTCTTTTCTGGTCGACTCCGCATTGACCTGATCCTGCTgtaagtacccattcagcCtaa gcaaagttccggacttgaactaataaatcatctcagGTACTCCTTCTGTCGCCTGGCTGATGATCTAGTCGACGACGCCA AATCTCGCCGTGAGGTCTTGTCCTGGACCGCGAAGCTGAACCACTTCCTTGATCTGCACTACAAGGACGCGGACGCCACC GAGGACCCCAAGAAAAAGGCGGAGCGAATCGACGCCTACATCAAGACAGCGTTCCCTCCCTGTGCCTACCAAGCCCTCCA ССTСCTGCCСАСTCACATTCTTCCTCCCAAGCСTCTTTACGATCTCATCAAGGGTTTCGAGATGGACTCTCAATTCACCT TCCACGGTACTTCCGACTCTACGGATCTCCAATACCCCATCGCCGACGACAAGGACCTTGAGAACTACGCTATCTATGTC GCCGGTACCGTCGGCGAGCTCTGCATCGCCCTCATCATCTACCACTGCCTGCCAGACATGTCGGACACTCAGAAGCGCGA GCTCGAGACCGCCGCGTGCCGGATGGGCATCGCGCTGCAGTACGTCAACATCGCTCGTGACATCGTCGTCGACGCACGTA TCGGGCGCGTTTACTTGCCTACCACCTGGCTCAAGACAAGAAGGAAGGGTTGACGCACAAGATGGTCTTGGAGAACCCC GAGGGTCCCGAGGTCATTGAGCGGATGAGAAGACGGCTTTTGGAAAATGCGTTTGAGCTGTATGGGGGCGCGAGGCCTGA GATGCAACGGATACCGAGCGAGGCTAGGGGCCCGATGATTGGTGCCGTTGAAAATTACATGGCGATTGGAAGGGTGTTGA GGGAGAGGAAGGAGGGGACGGTGTTTGTGAGGATGGAGGGGAGGGCTACGGTCCCGAAGCGAAGGAGGTTGAGCACGCTG TTGAGGGCGTTGTATGAGCAGTAG 
FGSC900 (Accession No. FR745435)

Identical sequence in FGSC910

Replacement of $\mathrm{A}$ by $\underline{\mathrm{C}}$

ATGTACGACTATGCTTTTGTgtgagtaccagatctgacagccaccaccctttagtcctgggaacatcattgctgatga gatacatgatagTCACTTAAAATTCACGGTACCCGCGGCGGTACTTCTCACCGCTATCGCCTACCCCATTCTCAACAGGA TACATCTCATCCAAACAGGCTTCCTCGTCGTCGTCGCCTTTACCGCCGCTCTGCCATGGGATGCCTACTTGATTAAGCAC AAAGTATGGTCTTACCCACCAGAAGCCATTGTTGGGCCGCGTTTGCTTGGAATTCCCTTTGAAGAGCTGTTCTTCTTTGT GATACAGACTTACATCACGGCGCTCGTATACATCCTCTTCAACAAGCCGGTGCTGCACGCGTTGCACCTCAACAATCAAC AAAACCCGCCAGCATGGATGAGGGTTGTCAAGGTTACCGGCCAGGTAGTCCTCGTAGCCTTGTCGGTATGGGGATGGAAT GCCGCTCAGGTTCATCAGGAAACAAGCTATCTCGGCTTGATCCTTGTTTGGGCTTGTCCGTTCTTACTGGCTATCTGGAC ССTCGCTGGGCGCTTCATTCTCAGCCTACCCTGGTACGCGACGGTGCTCCCGATGTTCCTACCCACCTTCTATCTTTGGG CGGTAGACGAGTTTGCCTTGCACAGGGGTACTTGGTCCATCGGATCGGGGACGAAGCTCGATTTTTGTCTGTTTGGCAAG TTGGACATTGAAGAAGCCACGTTCTTCCTGGTGACCAACATGCTCATCGTTGGCGGTATGGCCGCGTTCGATCAATATCT GGCCGTCATTTACGCTTTCCCAACTCTGTTCCCCAAGGTCAACCGGTATCCGACAACTCATATGCTTCTTCAAAGCCGTC TTATCAACACTTCCAGGTACGATCTTGAGCGCATTGAGGGCCTGAGAGAAGCGGTCGAGAGACTGCGCCTGAAGAGCAGG AGTTTTTACCTGGCCAATTCGCTCTTTTCTGGTCGACTCCGCATTGACCTGATCCTGCTgtaagtacccattcagcCtaa gcaagttccggacttgaactaataatcatctcagGTCCTCCTTCTGTCGCCTGGCTGATGATCTAGTCGACGACGCCA АATCTCGCCGTGAGGTCTTGTCCTGGACCGCGAAGCTGÄACCACTTCCTTGATCTGCACTACAAGGACGCGGACGCCACC GAGGACCCCAAGAAAAAGGCGGAGCGAATCGACGCCTACATCAAGACAGCGTTCCСTCCCTGTGCCTACCAAGCCCTCCA ССTCCTGCCCACTCACATTCTTCCTCCCAAGCCTCTTTACGATCTCATCAAGGGTTTCGAGATGGACTCTCAATTCACCT TCCACGGTACTTCCGACTCTACGGATCTCCAATACCCCATCGCCGACGACAAGGACCTTGAGAACTACGCTATCTATGTC GCCGGTACCGTCGGCGAGCTCTGCATCGCCCTCATCATCTACCACTGCCTGCCAGACATGTCGGACACTCAGAAGCGCGA GCTCGAGACCGCCGCGTGCCGGATGGGCATCGCGCTGCAGTACGTCAACATCGCTCGTGACATCGTCGTCGACGCACGTA TCGGGCGCGTTTACTTGCCTACCACCTGGCTCAAGAAGGAAGGGTTGACGCACAAGATGGTCTTGGAGAACCCCGAGGGT CCCGAGGTCATTGAGCGGATGAGAAGACGGCTTTTGGAAAATGCGTTTGAGCTGTATGGGGGCGCGAGGCCTGAGATGCA ACGGATACCGAGCGAGGCTAGGGGCCCGATGATTGGTGCCGTTGAAAATTACATGGCGATTGGAAGGGTGTTGAGGGAGA GGAAGGAGGGGACGGTGTTTGTGAGGATGGAGGGGAGGGCTACGGTCCCGAAGCGAAGGAGGTTGAGCACGCTGTTGAGG GCGTTGTATGAGCAGTAG

FGSC904 (Accession No. FR745432)

Replacement of GAA by AG

ATGTACGACTATGCTTTTGTgtgagtaccagatctgacagccaccaccctttagtcctgggaaacatcattgctgatga gatacatgatagTCACTTAAAATTCACGGTACCCGCGGCGGTACTTCTCACCGCTATCGCCTACCCCATTCTCAACAGGA TACATCTCATCCAAACAGGCTTCCTCGTCGTCGTCGCCTTTACCGCCGCTCTGCCATGGGATGCCTACTTGATTAAGCAC AAAGTATGGTCTTACCCACCAGAAGCCATTGTTGGGCCGCGTTTGCTTGGAATTCCCTTTGAAGAGCTGTTCTTCTTTGT GATACAGACTTACATCACGGCGCTCGTATACATCCTCTTCAACAAGCCGGTGCTGCACGCGTTGCACCTCAACAATCAAC AAAACCCGCCAGCATGGATGAGGGTTGTCAAGGTTACCGGCCAGGTAGTCCTCGTAGCCTTGTCGGTATGGGGATGGAAT GCCGCTCAGGTTCATCAGGAAACAAGCTATCTCGGCTTGATCCTTGTTTGGGCTTGTCCGTTCTTACTGGCTATCTGGAC ССTCGCTGGGCGCTTCATTCTCAGCCTACCCTGGTACGCGACGGTGCTCCCGATGTTCCTACCCACCTTCTATCTTTGGG CGGTAGACGAGTTTGCCTTGCACAGGGGTACTTGGTCCATCGGATCGGGGACGAAGCTCGATTTTTGTCTGTTTGGCAAG TTGGACATTGAAGAAGCCACGTTCTTCCTGGTGACCAACATGCTCATCGTTGGCGGTATGGCCGCGTTCGATCAATATCT GGCCGTCATTTACGCTTTCCCAACTCTGTTCCCCAAGGTCAACCGGTATCCGACAACTCATATGCTTCTTCAAAGCCGTC TTATCAACACTTCCAGGTACGATCTTGAGCGCATTGAGGGCCTGAGAGAAGCGGTCGAGAGACTGCGCCTGAAGAGCAGG AGTTTTTACCTGGCCAATTCGCTCTTTTCTGGTCGACTCCGCATTGACCTGATCCTGCTgtaagtacccattcagcCtaa gcaaagttccggacttgaactaataatcatctcagGTACTCCTTCTGTCGCCTGGCTGATGATCTAGTCGACGACGCCA AATCTCGCCGTGAGGTCTTGTCCTGGACCGCGAAGCTGAACCACTTCCTTGATCTGCACTACAAGGACGCGGACGCCACC GAGGACCCCAAAGAAAGGCGGAGCGAATCGACGCCTACATCAAGACAGCGTTCCCTCCCTGTGCCTACCAAGCCCTCCAC СTCСTGCCСАСTCACATTCTTCСTCCCAAGCСTCTTTACGATCTCATCAAGGGTTTCGAGATGGACTCTCAATTCACCTT CCACGGTACTTCCGACTCTACGGATCTCCAATACCCCATCGCCGACGACAAGGACCTTGAGAACTACGCTATCTATGTCG CCGGTACCGTCGGCGAGCTCTGCATCGCCCTCATCATCTACCACTGCCTGCCAGACATGTCGGACACTCAGAAGCGCGAG CTCGAGACCGCCGCGTGCCGGATGGGCATCGCGCTGCAGTACGTCAACATCGCTCGTGACATCGTCGTCGACGCACGTAT CGGGCGCGTTTACTTGCCTACCACCTGGCTCAAGAAGGAAGGGTTGACGCACAAGATGGTCTTGGAGAACCCCGAGGGT CCCGAGGTCATTGAGCGGATGAGAAGACGGCTTTTGGAAAATGCGTTTGAGCTGTATGGGGGCGCGAGGCCTGAGATGCA ACGGATACCGAGCGAGGCTAGGGGCCCGATGATTGGTGCCGTTGAAAATTACATGGCGATTGGAAGGGTGTTGAGGGAGA GGAAGGAGGGGACGGTGTTTGTGAGGATGGAGGGGAGGGCTACGGTCCCGAAGCGAAGGAGGTTGAGCACGCTGTTGAGG GCGTTGTATGAGCAGTAG 
FGSC913 (Accession No. FR745433)

Deletion of $(\underline{T})$

ATGTACGACTATGCTTTTGTgtgagtaccagatctgacagccaccaccctttagtcctgggaacatcattgctgatga gatacatgatagTCACTTAAAATTCACGGTACCCGCGGCGGTACTTCTCACCGCTATCGCCTACCCCATTCTCAACAGGA TACATCTCATCCAAACAGGCTTCCTCGTCGTCGTCGCCTTTACCGCCGCTCTGCCATGGGATGCCTACTTGATTAAGCAC AAAGTATGGTCTTACCCACCAGAAGCCATTGTTGGGCCGCGTTTGCTTGGAATTCCCTTTGAAGAGCTGTTCTTCTTTGT GATACAGACTTACATCACGGCGCTCGTATACATCCTCTTCAACAAGCCGGTGCTGCACGCGTTGCACCTCAACAATCAAC AAAACCCGCCAGCATGGATGAGGGTTGTCAAGGTTACCGGCCAGGTAGTCCTCGTAGCCTTGTCGGTATGGGGATGGAAT GCCGCTCAGGTTCATCAGGAAACAAGCTATCTCGGCTTGATCCTTGTTTGGGCTTGTCCGTTCTTACTGGCTATCTGGAC ССTCGCTGGGCGCTTCATTCTCAGCCTACCCTGGTACGCGACGGTGCTCCCGATGTTCCTACCCACCTTCTATCTTTGGG CGGTAGACGAGTTTGCCTTGCACAGGGGTACTTGGTCCATCGGATCGGGGACGAAGCTCGATTTTTGTCTGTTTGGCAAG TTGGACATTGAAGAAGCCACGTTCTTCCTGGTGACCAACATGCTCATCGTTGGCGGTATGGCCGCGTTCGATCAATATCT GGCCGTCATTTACGCTTTCCCAACTCTGTTCCCCAAGGTCAACCGGTATCCGACAACTCATATGCTTCTTCAAAGCCGTC TTATCAACACTTCCAGGTACGATCTTGAGCGCATTGAGGGCCTGAGAGAAGCGGTCGAGAGACTGCGCCTGAAGAGCAGG AGTTTTTACCTGGCCAATTCGCTCTTTTCTGGTCGACTCCGCATTGACCTGATCCTGCTgtaagtaccCattcagcctaa gcaagttccggacttgaactaataatcatctcagGTACTCCTTCTGTCGCCTGGCTGATGATCTAGTCGACGACGCCA AATCTCGCCGTGAGGTCTTGTCCTGGACCGCGAAGCTGAACCACTTCCTTGATCTGCACTACAAGGACGCGGACGCCACC GAGGACCCCAAGAAAAAGGCGGAGCGAATCGACGCC ( T) ACATCAAGACAGCGTTCCCTCCCTGTGCCTACCAAGCCCTC САССTCCTGCCСАСTCACATTCTTCСTCCCAAGCCTCTTTACGATCTCATCAAGGGTTTCGAGATGGACTCTCAATTCAC СTTCCACGGTACTTCCGACTCTACGGATCTCCAATACCCCATCGCCGACGACAAGGACCTTGAGAACTACGCTATCTATG TCGCCGGTACCGTCGGCGAGCTCTGCATCGCCCTCATCATCTACCACTGCCTGCCAGACATGTCGGACACTCAGAAGCGC GAGCTCGAGACCGCCGCGTGCCGGATGGGCATCGCGCTGCAGTACGTCAACATCGCTCGTGACATCGTCGTCGACGCACG TATCGGGCGCGTTTACTTGCCTACCACCTGGCTCAAGAAGGAAGGGTTGACGCACAAGATGGTCTTGGAGAACCCCGAG GGTCCCGAGGTCATTGAGCGGATGAGAAGACGGCTTTTGGAAAATGCGTTTGAGCTGTATGGGGGCGCGAGGCCTGAGAT GCAACGGATACCGAGCGAGGCTAGGGGCCCGATGATTGGTGCCGTTGAAAATTACATGGCGATTGGAAGGGTGTTGAGGG AGAGGAAGGAGGGGACGGTGTTTGTGAGGATGGAGGGGAGGGCTACGGTCCCGAAGCGAAGGAGGTTGAGCACGCTGTTG AGGGCGTTGTATGAGCAGTAG

FGSC914 (Accession No. FR745434)

Replacement of $\mathrm{G}$ by $\underline{\mathrm{A}}$

ATGTACGACTATGCTTTTGTgtgagtaccagatctgacagccaccaccctttagtcctgggaacatcattgctgatga gatacatgatagTCACTTAAAATTCACGGTACCCGCGGCGGTACTTCTCACCGCTATCGCCTACCCCATTCTCAACAGGA TACATCTCATCCAAACAGGCTTCCTCGTCGTCGTCGCCTTTACCGCCGCTCTGCCATGGGATGCCTACTTGATTAAGCAC AAAGTATGGTCTTACCCACCAGAAGCCATTGTTGGGCCGCGTTTGCTTGGAATTCCCTTTGAAGAGCTGTTCTTCTTTGT GATACAGACTTACATCACGGCGCTCGTATACATCCTCTTCAACAAGCCGGTGCTGCACGCGTTGCACCTCAACAATCAAC AAAACCCGCCAGCATGGATGAGGGTTGTCAAGGTTACCGGCCAGGTAGTCCTCGTAGCCTTGTCGGTATGGGGATGGAAT GCCGCTCAGGTTCATCAGGAAACAAGCTATCTCGGCTTGATCCTTGTTTGGGCTTGTCCGTTCTTACTGGCTATCTGGAC ССTCGCTGGGCGCTTCATTCTCAGCCTACCCTGGTACGCGACGGTGCTCCCGATGTTCCTACCCACCTTCTATCTTTGGG CGGTAGACGAGTTTGCCTTGCACAGGGGTACTTGGTCCATCGGATCGGGGACGAAGCTCGATTTTTGTCTGTTTGGCAAG TTGGACATTGAAGAAGCCACGTTCTTCCTGGTGACCAACATGCTCATCGTTGGCGGTATGGCCGCGTTCGATCAATATCT GGCCGTCATTTACGCTTTCCCAACTCTGTTCCCCAAGGTCAACCGGTATCCGACAACTCATATGCTTCTTCAAAGCCGTC TTATCAACACTTCCAGGTACGATCTTGAGCGCATTGAGGGCCTGAGAGAAGCGGTCGAGAGACTGCGCCTGAAGAGCAGG AGTTTTTACCTGGCCAATTCGCTCTTTTCTGGTCGACTCCGCATTGACCTGATCCTGCTgtaagtacccattcagcctaa gcaagttccggacttgaactaataatcatctcagGTACTCCTTCTGTCGCCTGGCTGATGATCTAGTCGACGACGCCA AATCTCGCCGTGAGGTCTTGTCCTGAACCGCGAAGCTGAACCACTTCCTTGATCTGCACTACAAGGACGCGGACGCCAC CGAGGACCCCAAGAAAAAGGCGGAGCGAATCGACGCCTACATCAAGACAGCGTTCCCTCCCTGTGCCTACCAAGCCCTCC ACСTCСTGCCСАСTCACATTCTTCСTCCCAAGCСTCTTTACGATCTCATCAAGGGTTTCGAGATGGACTCTCAATTCACC TTCCACGGTACTTCCGACTCTACGGATCTCCAATACCCCATCGCCGACGACAAGGACCTTGAGAACTACGCTATCTATGT CGCCGGTACCGTCGGCGAGCTCTGCATCGCCCTCATCATCTACCACTGCCTGCCAGACATGTCGGACACTCAGAAGCGCG AGCTCGAGACCGCCGCGTGCCGGATGGGCATCGCGCTGCAGTACGTCAACATCGCTCGTGACATCGTCGTCGACGCACGT ATCGGGCGCGTTTACTTGCCTACCACCTGGCTCAAGAAGGAAGGGTTGACGCACAAGATGGTCTTGGAGAACCCCGAGGG TCCCGAGGTCATTGAGCGGATGAGAAGACGGCTTTTGGAAAATGCGTTTGAGCTGTATGGGGGCGCGAGGCCTGAGATGC AACGGATACCGAGCGAGGCTAGGGGCCCGATGATTGGTGCCGTTGAAAATTACATGGCGATTGGAAGGGTGTTGAGGGAG AGGAAGGAGGGGACGGTGTTTGTGAGGATGGAGGGGAGGGCTACGGTCCCGAAGCGAAGGAGGTTGAGCACGCTGTTGAG GGCGTTGTATGAGCAGTAG 
FGSC2666 (Accession No. FR745436)

19 point mutations; silent mutations in yellow

ATGTACGACTATGCTTTTGTgtgagtaccagatctgacagccaccaccctttagtcctgggaaacatcattgctgatga gatacatgatagTCACTTAAAATTCACGGTACCCGTGGCGGTACTTCTCACCGCTATCGCCTACCCCATTCTCAACAGGA TACATCTCATCCAAACAGGCTTCCTCGTCGTCGTCGCCTTTACCGCCGCTCTGCCATGGGATGCCTACTTGATTAAGCAC AAAGTATGGTCTGACCCACCAGAAGCCATTGTTGGGCCGCGTTTGCTTGGAATTCCCTTTGAAGAGCTGTTCTTCTTTGT GATACAGACTTACATCACGGCGCTCGTATACATCCTCTTCAACAAGCCGGTGCTGCACGCGTTGCACCTCAACAATCAAC AAAACCCGCCAGCGTGGATGAGGGTTGTCAAGGTTACCGGTCAGGTAGTCCTCGTAGCCTTGTCGGTATGGGGATGGAAT GCCGCTCAGGTTCATCAGGAAACAAGCTATCTCGGCTTGATCCTTGTTTGGGCTTGTCCGTTCTTACTGGCTATCTGGAC ССTCGCTGGGCGCTTCATTCTCAGCCTACCATGGTACGCGACGCTGCTCCCGATGTTCCTACCCACCTTCTATCTTTGGG CGGTAGACGAGTTTGCCTTGCACAGGGGTACTTGGTCCATCGGATCGGGGACGAAGCTCGATTTTTGTCTGTTTGGCAAG TTGGACATTGAAGAAGCCACGTTCTTCCTGGTGACCAACATGCTCATCGTTGGCGGTATGGCCGCGTTCGATCAATACCT GGCCGTCATTTACGCTTTCCCAACGCTGTTCCCCAAGGTCAACCGGTATCCGACACCTCATATGCTTCTTCAAAGCCGTC TTATCAACACTTCCAGGTACGATCTTGAGCGCATTGAGGGTCTGAGAGAAGCGGTCGAGAGACTGCGCCTGAAGAGCAGG AGTTTTTACCTGGCCAATTCGCTCTTTTCTGGTCGACTCCGCATTGACCTGATCCTGCTgtaagtacccattcagcctaa ccaagttccggacttgaactaataaaccatctcagGTACTCCTTCTGTCGCCTGGCTGATGATCTAGTCGACGACGCTA ӒТTCTCCCGCGAGGTCTTGTCCTGGACCGCGAAGCTGAACCACTTCCTTGATCTGCACTACAAGGACGCGGACGCCACC GAGGACCCCAÄGAAAAAGGCGGAGCGAATCGACGCCTACATCAAGACAGCGTTCCCTCCCTGTGCCTACCAAGCCCTCCA ССTCCTGCCCACTCACATTCTTCCTCCCAAGCCTCTTTACGATCTCATCAAGGGTTTCGAGATGGACTCTCAATTCACCT TCCACGGTACTTCTGACTCTACGGATCTCCAATACCCCATCGCCGACGACAAGGACCTTGAGAACTACGCTATCTATGTC GCCGGCACCGTCGĞCGAGCTCTGCATCGCCCTCATCATCTACCACTGCCTGCCAGACATGTCGGACACTCAGAAGCGCGA GCTCGÄGACCGCCGCGTGCCGGATGGGCATTGCGCTGCAGTACGTCAACATCGCTCGCGACATCGTCGTCGACGCACGTA TTGGGCGCGTTTACTTGCCTACCACCTGGCTCAAGAAGGAAGGGTTGACGCACAAGATGGTCTTGGAGAACCCCGAGGGT C̄CCAGGTCATTGAGCGGATGAGAAGACGGCTTTTGGAAAATGCGTTTGAGCTGTATGGGGGCGCGAGGCCTGAGATGCA ACGGATACCGAGCGAGGCTAGGGGCCCGATGATTGGTGCCGAGGAAAATTACATGGCGATTGGAAGGGTGTTGAGGGAGA GGAAGGAGGGGACGGTGTTTGTGAGGATGGAGGGGAGGGCTACGGTCCCGAAGCGAAGGAGGTTGAGCACGCTGTTGAGG GCGTTGTATGAGCAGTAG

FGSC4014 (Accession No. FR745437)

11 point mutations; silent mutations in yellow

ATGTACGACTATGCTTTTGTgtgagtaccagatctgacagccaccaccctttagtcctgggaacatcattgctgatga gatacatgatagTCACTTAAAATTCACGGTACCCGCGGCGGTACTTCTCACCGCTATCGCCTACCCCATTCTCAACAGGA TACATCTCATCCAAACAGGCTTCCTCGTCGTCGTCGCCTTTACCGCCGCTCTGCCATGGGATGCCTACTTGATTAAGCAC AAAGTATGGTCTTACCCACCAGAAGCCATTGTTGGGCCGCGTTTGCTTGGAATTCCCTTTGAAGAGCTGTTCTTCTTTGT GATACAGACTTACATCACGGCGCTCGTATACATCCTCTTCAACAAGCCGGTGCTGCACGCGTTGCACCTCAACAATCAAC AAAACCCGCCAGCATGGATGAGGGTTGTCAAGGTTACCGGCCAGGTAGTCCTCGTAGCCTTGTCGGTATGGGGATGGAAT GCCGCTCAGGTTCATCAGGAAACAAGCTATCTCGGCTTGATCCTTGTTTGGGCTTGTCCGTTCTTACTGGCTATCTGGAC ССTCGCTGGGCGCTTCATTCTCAGCCTACCCTGGTACGCGACGGTGCTCCCGATGTTCCTACCCACCTTCTATCTTTGGG CGGTAGACGAGTTTGCCTTGCACAGGGGTACTTGGTCCATCGGATCGGGGACGAAGCTCGATTTTTGTCTGTTTGGCAAG TTGGACATTGAAGAAGCCACGTTCTTCCTGGTGACCAACATGCTCATCGTTGGCGGTATGGCCGCGTTCGATCAATATCT GGCCGTCATTTACGCTTTCCCAACTCTGTTCCCCAAGGTCAACCGGTATCCGACAGCTCATATGCTTCTTCAAAGCCGTC TTATCAACACTTCCAGGTACGATCTTGAGCGCATTGAGGGTCTGAGAGAAGCGGTCGAGAGACTGCGCCTGAAGAGCAGG AGTTTTTACCTGGCCAATTCGCTCTTTTCTGGTCGACTCCGCATTGACCTGATCCTGCTgtaagtaccCattcagcctaa ccaagttccggacttgaactaataatcatctcagGTACTCCTTCTGTCGCCTGGCTGATGATCTAGTCGACGACGCTA AАTCTCGCCGCGAGGTCTTGTCCTGGACCGCGAAGCTGAACCACTTCCTTGATCTGCACTACAAGGACGCGGACGCCACC GAGGACCCCAÄGAAAAAGGCGGAGCGAATCGACGCCTACATCAAGACAGCGTTCCCTCCCTGTGCCTACCAAGCCCTCCA ССTCCTGCCCACTCACATTCTTCCTCCCAAGCCTCTTTACGATCTCATCAAGGGTTTCGAGATGGACTCTCAATTCACCT TCCACGGTACTTCTGACTCTACGGATCTCCAATACCCCATCGCCGACGACAAGGACCTTGAGAACTACGCTATCTATGTC GCCGGCACCGTCGGCGAGCTCTGCATCGCCCTCATCATCTACCACTGCCTGCCAGACATGTCGGACACTCAGAAGCGCGA GCTCGÄGACCGCCGCGTGCCGGATGGGCATTGCGCTGCAGTACGTCAACATCGCTCGCGACATCGTCGTCGACGCACGTA TTGGGCGCGTTTACTTGCCTACCACCTGGCTCAAGAAGGAAGGGTTGACGCACAAGATGGTCTTGGAGAACCCCGAGGGT CDCGAGGTCATTGAGCGGATGAGAAGACGGCTTTTGGAAAATGCGTTTGAGCTGTATGGGGGCGCGAGGCCTGAGATGCA ACGGATACCGAGCGAGGCTAGGGGCCCGATGATTGGTGCCGAGGAAAATTACATGGCGATTGGAAGGGTGTTGAGGGAGA GGAAGGAGGGGACGGTGTTTGTGAGGATGGAGGGGAGGGCTACGGTCCCGAAGCGAAGGAGGTTGAGCACGCTGTTGAGG GCGTTGTATGAGCAGTAG 